BULLETIN OF THE

AMERICAN MATHEMATICAL SOCIETY

Volume 77, Number 6, November 1971

\title{
PFISTER FORMS AND $K$-THEORY OF FIELDS ${ }^{1}$
}

\author{
BY RICHARD ELMAN AND T. Y. LAM
}

Communicated by Hyman Bass, May 20, 1971

Let $F$ be a field of characteristic different from two. We shall write $W(F)$ to denote the Witt ring of $F$, and $I(F)$ to denote the ideal of all even dimensional forms in $W(F)$. Also, let $K_{n} F(n \geqq 1)$ be the higher $K$-groups of $F$ defined by Milnor in [3], and $k_{n} F=K_{n} F / 2 K_{n} F$. The elements $l\left(a_{1}\right) \cdots l\left(a_{n}\right) \in k_{n} F$ will be called the generators of $k_{n} F$. If $\left(a_{1}, \cdots, a_{n}\right)$ is an $n$-tuple of nonzero elements of $F$, we shall write $\left\langle\left\langle a_{1}, \cdots, a_{n}\right\rangle\right\rangle$ for the $2^{n}$-dimensional quadratic form $\bigotimes_{i=1}^{n}\left\langle a_{i}, 1\right\rangle$, and refer to it as an $n$-fold Pfister form. Clearly, these $n$-fold Pfister forms give a system of generators of $I^{n} F$ as an ideal in $W(F)$. In [3], Milnor showed that

$$
s_{n}\left(l\left(a_{1}\right) \cdots l\left(a_{n}\right)\right)=\left\langle\left\langle-a_{1}, \cdots,-a_{n}\right\rangle\right\rangle\left(\bmod I^{n+1} F\right)
$$

gives a well-defined epimorphism from $k_{n} F$ onto $I^{n} F / I^{n+1} F$. In $\S 4$ of [3], Milnor raised the question whether these maps are isomorphisms.

In studying this problem, the Pfister forms clearly play a crucial role. In this note, we announce the following results.

TheOREM 1. The following statements are equivalent:

(1) $\left\langle\left\langle-a_{1},-a_{2}\right\rangle\right\rangle,\left\langle\left\langle-b_{1},-b_{2}\right\rangle\right\rangle$ are isometric (Pfister) forms.

(2) $\left\langle\left\langle-a_{1},-a_{2}\right\rangle\right\rangle \equiv\left\langle\left\langle-b_{1},-b_{2}\right\rangle\right\rangle\left(\bmod I^{3} F\right)$.

(3) $\left(\frac{a_{1}, a_{2}}{F}\right),\left(\frac{b_{1}, b_{2}}{F}\right)$ are isomorphic (quaternion) algebras.

(4) $l\left(a_{1}\right) l\left(a_{2}\right)=l\left(b_{1}\right) l\left(b_{2}\right)$ in $k_{2} F$.

This theorem shows that $l\left(a_{1}\right) l\left(a_{2}\right) \in k_{2} F$ or the quaternion algebra

$$
\left(\frac{a_{1}, a_{2}}{F}\right)
$$

(in the Brauer group of $F$ ) gives a complete invariant for the isometry class of a 2 -fold Pfister form $\left\langle\left\langle-a_{1},-a_{2}\right\rangle\right\rangle$. One therefore naturally asks if an analogous result will hold for the isometry class of an

AMS 1970 subject classifications. Primary 15A63, 18F25; Secondary 13D15.

Key words and phrases. Quadratic forms, Pfister forms, Witt rings, isometry, algebraic $K$-theory.

${ }^{1}$ Partially supported by NSF GP-20532.

Copyright (c) American Mathematical Society 1971 
$n$-fold Pfister form. Motivated by this, one is led to the following definition. Let $\left\langle\left\langle a_{1}, \cdots, a_{n}\right\rangle\right\rangle$ and $\left\langle\left\langle b_{1}, \cdots, b_{n}\right\rangle\right\rangle$ be two $n$-fold Pfister forms $(n \geqq 2)$. We shall say that they are simply-p-equivalent if there exist two distinct indices $i$ and $j$ such that

(a) $\left\langle\left\langle a_{i}, a_{j}\right\rangle\right\rangle \cong\left\langle\left\langle b_{i}, b_{j}\right\rangle\right\rangle$ (see Theorem 1 for equivalent conditions), and

(b) $a_{k}=b_{k}$ whenever $k$ is different from $i$ and $j$.

More generally, we say that two $n$-fold Pfister forms $\phi$ and $\phi^{\prime}$ are chain-p-equivalent if there exists a sequence of $n$-fold Pfister forms $\phi_{0}, \phi_{1}, \cdots, \phi_{m}$ such that $\phi_{0}=\phi, \phi_{m}=\phi^{\prime}$ and that each $\phi_{i}$ is simply-pequivalent to $\phi_{i+1}(0 \leqq i \leqq m-1)$. This is clearly an equivalence relation on all $n$-fold Pfister forms, and Theorem 1 shows if $\left\langle\left\langle-a_{1}, \cdots,-a_{n}\right\rangle\right\rangle$ is chain- $p$-equivalent to $\left\langle\left\langle-b_{1}, \cdots,-b_{n}\right\rangle\right\rangle$, then $l\left(a_{1}\right) \cdots l\left(a_{n}\right)$ equals $l\left(b_{1}\right) \cdots l\left(b_{n}\right)$ in $k_{n} F$. Further, it turns out that chain-p-equivalence of $n$-fold Pfister forms coincides with ordinary isometry of such forms. This may be viewed as an analog of Witt's classical chain equivalence theorem [5].

Theorem 1 now generalizes as follows:

THEOREM 2. The following statements are equivalent:

(1) $\left\langle\left\langle-a_{1}, \cdots,-a_{n}\right\rangle\right\rangle$ and $\left\langle\left\langle-b_{1}, \cdots,-b_{n}\right\rangle\right\rangle$ are chain-pequivalent.

(2) $l\left(a_{1}\right) \cdots l\left(a_{n}\right)=l\left(b_{1}\right) \cdots l\left(b_{n}\right)$ in $k_{n} F$.

(3) $\left\langle\left\langle-a_{1}, \cdots,-a_{n}\right\rangle\right\rangle \equiv\left\langle\left\langle-b_{1}, \cdots,-b_{n}\right\rangle\right\rangle\left(\bmod I^{n+1} F\right)$.

(4) $\left\langle\left\langle-a_{1}, \cdots,-a_{n}\right\rangle\right\rangle$ and $\left\langle\left\langle-b_{1}, \cdots,-b_{n}\right\rangle\right\rangle$ are isometric.

(5) There exist nonzero elements $a$ and $b$ in $F$ such that $\langle a\rangle \otimes\left\langle\left\langle-a_{1}\right.\right.$, $\left.\left.\cdots,-a_{n}\right\rangle\right\rangle$ is isometric to $\langle b\rangle \otimes\left\langle\left\langle-b_{1}, \cdots,-b_{n}\right\rangle\right\rangle$.

In particular $l\left(a_{1}\right) l\left(a_{2}\right) \cdots l\left(a_{n}\right) \in k_{n} F$ is a complete invariant for the isometry class of a Pfister form $\left\langle\left\langle-a_{1}, \cdots,-a_{n}\right\rangle\right\rangle$.

COROLlary 1. $\left\langle\left\langle-a_{1}, \cdots,-a_{n}\right\rangle\right\rangle$ is hyperbolic if and only if $l\left(a_{1}\right) \cdots l\left(a_{n}\right)=0$ in $k_{n} F$. In particular $k_{n} F=0$ if and only if $I^{n} F=0$, if and only if every $n$-fold Pfister form is hyperbolic.

Corollary 2. If the level of $F$ is $s=2^{m}$, then $m$ is precisely the smallest integer such that $l(-1)^{m+1}=0$ in $k_{m+1} F$. (This refines Milnor's result in [3, Theorem 1.4].) Furthermore, $\operatorname{dim}_{z_{2}} k_{m-r+1} F \geqq r(r+1) / 2(1 \leqq r \leqq m)$, and $|W(F)| \geqq 2 \cdot 2^{m(m+1)(m+2) / 6}$.

Theorem 2 is proved by showing the chain of implications $(1) \Rightarrow(2)$ $\Rightarrow(3) \Rightarrow(4) \Rightarrow(1)((4)$ and (5) are easily seen to be equivalent). The implication $(3) \Rightarrow(4)$ is proved by extending the techniques of Arason 
and Pfister [1], while (4) $\Rightarrow(1)$ is based on the following Proposition.

Proposition. If $\tau=\left\langle\left\langle a_{1}, \cdots, a_{r}\right\rangle\right\rangle$ and $\sigma=\left\langle\left\langle b_{1}, \cdots, b_{s}\right\rangle\right\rangle=\langle 1\rangle \perp \sigma^{\prime}$, and if $c_{1} \neq 0$ is represented by the form $\tau \otimes \sigma^{\prime}$, then there exist nonzero $c_{2}, \cdots, c_{s}$ in $F$ such that $=\left\langle\left\langle a_{1}, \cdots, a_{r}, b_{1}, \cdots, b_{s}\right\rangle\right\rangle$ is chain-pequivalent to $\left\langle\left\langle a_{1}, \cdots, a_{r}, c_{1}, \cdots, c_{s}\right\rangle\right\rangle$.

Note that the Proposition also gives a proof of the following wellknown fact: if a $k$-fold Pfister form $\phi_{1}$ is a subform of an $n$-fold Pfister form $\gamma$, then there exists an $(n-k)$-fold Pfister form $\phi_{2}$ such that $\gamma \cong \phi_{1} \otimes \phi_{2}$. The known proof of this utilizes Pfister's theory of strongly multiplicative forms (see [4]).

The Proposition also yields the following:

Theorem 3. Suppose $\phi, \tau$ are $n$-fold Pfister forms. Then $\gamma=$ $\phi \perp\langle-1\rangle \tau$ contains a hyperbolic form $2^{r} H(r \leqq n$ and $H$ denotes the hyperbolic plane $\langle 1,-1\rangle)$ if and only if there exist an $r$-fold Pfister form $\sigma$ and two $(n-r)$-fold Pfister forms $\phi_{0}$ and $\tau_{0}$ such that $\phi \cong \sigma \otimes \phi_{0}$ and $\tau \cong \sigma \otimes \tau_{0}$. Furthermore the Witt index of $\gamma$ is a 2-power.

Corollary 1. Suppose every $2^{n}$-dimensional form over $F$ is universal. Then

(1) $I^{n+1} F=0$;

(2) $s_{n}$ is an isomorphism from $k_{n} F$ to $I^{n} F$;

(3) every element of $I^{n} F$ is represented by an $n$-fold Pfister form;

(4) every pair of $n$-fold Pfister forms $\phi, \tau$ can be written as $\sigma \otimes \phi_{0}$ and $\sigma \otimes \tau_{0}$, where $\sigma$ is an $(n-1)$-fold Pfister form, and $\phi_{0}, \tau_{0}$ are 1-fold Pfister forms.

Corollary 2. If $F$ is a $C_{3}$-field (e.g. any function field of transcendence degree 3 over the complex numbers), then for all $n, s_{n}: k_{n} \rightarrow I^{n} / I^{n+1}$ is an isomorphism for $F$, and hence also for $F(X)$, by [3, Corollary 5.8].

Using Theorems 2 and 3, we also obtain the following:

THEOREM 4. Let $\phi_{i}=\left\langle\left\langle a_{i 1}, \cdots, a_{i n}\right\rangle\right\rangle(i=1,2,3)$ be three $n$-fold Pfister forms. If $\phi_{1} \perp \phi_{2} \perp \phi_{3} \in I^{n+1}(F)$, then there exist an $(n-1)$-fold Pfister form $\tau$ and nonzero elements $x, y$ in $F$, such that

$$
\phi_{1} \cong \tau \otimes\langle-x y, 1\rangle, \quad \phi_{2} \cong \tau \otimes\langle x, 1\rangle, \quad \text { and } \phi_{3} \cong \tau \otimes\langle y, 1\rangle .
$$

In particular, there is an isometry $\langle-y\rangle \phi_{1} \perp \phi_{3} \cong \phi_{2} \perp 2^{n-1} H$. Also, the summation $\sum_{i=1}^{3} l\left(-a_{i 1}\right) \cdots l\left(-a_{i n}\right)$ vanishes in $k_{n} F$.

CoROllary 1. If every element in $k_{n} F$ can be expressed as a sum of at most three generators, then $s_{n}: k_{n} F \rightarrow I^{n} F / I^{n+1} F$ is an isomorphism. In particular, if $\left|k_{n} F\right| \leqq 64$, then $s_{n}$ is an isomorphism. 
CoROllary 2. Let $n$ be a fixed integer, and suppose that every element of $k_{n} F$ is equal to a generator, then every element in $k_{m} F(m \geqq n)$ is also equal to a generator of $k_{m} F$. In particular, $s_{m}: k_{m} F \rightarrow I^{m} F / I^{m+1} F$ is an isomorphism for all $m \geqq n$. Furthermore, given any pair of $m$-fold Pfister forms $\phi$ and $\tau(m \geqq n)$, there exist an $(m-1)$-fold Pfister form $\sigma$ and nonzero elements $x, y$ such that $\phi \cong \sigma \otimes\langle x, 1\rangle$ and $\tau \cong \sigma \otimes\langle y, 1\rangle$.

We will demonstrate the usefulness of Corollary 2 by two examples:

EXAmple 1. Suppose $k_{2} F$ has at most four elements. Then one can show: every element of $k_{2} F$ is equal to a generator (in which case Corollary 2 applies).

ExAmple 2. Let $F$ be a global field. Then one can show that every element of $k_{3} F$ is equal to a generator (see [3, Appendix], or [2]). Hence the corollary also applies in this case. Actually, every element of $k_{2} F$ is equal to a generator.

In these and some other examples, we have been able to determine all relations among Pfister forms.

We wish to express our hearty thanks to Arason and Pfister, who sent us the preprint of their work [1], from which some of the ideas in this note developed.

\section{REFERENCES}

1. J. K. Arason and A. Pfister, Beweis des Krullschen Durchschnittsatzes fiir den Wittring (to appear).

2. R. Elman and T. Y. Lam, Determination of $k_{n}(n \geqq 3)$ for global fields, Proc. Amer. Math. Soc. (to appear).

3. J. Milnor, Algebraic K-theory and quadratic forms, Invent. Math. 9 (1969/70), 318-344. MR 41 \#5465.

4. A. Pfister, Multiplikative quadratische Formen, Arch. Math. 16 (1965), 363-370. MR 32 \#2408.

5. E. Witt, Theorie der quadratischen Formen in beliebigen Körpern, J. Reine Angew. Math. 176 (1937), 31-44.

University of California, Berkeley, California 94720. 